\title{
Graded Visual Attention Modulates Brain Responses Evoked by Task-irrelevant Auditory Pitch Changes
}

\author{
Gunes Yucel ${ }^{1}$, Christopher Petty ${ }^{1}$, Gregory McCarthy ${ }^{1,2}$, \\ and Aysenil Belger ${ }^{1,3}$
}

\begin{abstract}
Previous studies suggested that auditory change-specific neural responses are attention-independent and reflect central auditory processing. The automaticity of the brain's response to infrequent changes in pitch within a series of auditory tone pips was examined in parallel functional magnetic resonance imaging (fMRI) and event-related potential (ERP) studies. Subjects performed a continuous perceptual-motor visual tracking task at two levels of difficulty while simultaneously hearing a series of task-irrelevant standard tone pips and infrequent pitch-deviant
\end{abstract}

\section{INTRODUCTION}

The information processing capacity of the brain is thought to be limited such that only a subset of the vast array of stimuli falling upon the body's sensors can be fully processed (Hillyard, Teder-Salejarvi, \& Munte, 1998). An organism's survival is thus dependent upon its ability to detect critical stimuli in the environment and to attend to those that are relevant to its current goals and needs. Increased attention to stimuli results in increased neural activity in stimulus-specific sensory cortical region, as demonstrated by recent studies of brain electrical activity (Alho, Woods, Algazi, \& Näätänen, 1992; Woods, Alho, \& Algazi, 1992) and functional brain imaging (Alho, Medvedev, et al., 1999; Woodruff, 1996). Similarly, behavioral and ERP studies have also suggested that spatial attention allocation enhances processing of information as a function of distance from the attended location (Arnott \& Alain, 2002; Spence, Ranson, \& Driver, 2000). Our interest here is the fate of sensory stimuli that fall outside of the current focus of attention-is there a corresponding decrease in the neural processing afforded to such stimuli? If so, an organism would be vulnerable if unattended stimuli presaged either survival-relevant threats or opportunities.

\footnotetext{
${ }^{1}$ Duke University Medical Center, Durham, NC, ${ }^{2}$ Department of Veterans Affairs Medical Center, Durham, NC, ${ }^{3}$ University of North Carolina at Chapel Hill
}

tones. fMRI results revealed that the unattended pitch-deviant tones strongly activated superior temporal and frontal cortical regions. These activations were significantly modulated by the tracking difficulty of the primary task. ERP results revealed that the amplitude of the scalp-negative component evoked by deviant tones (MMN) was attenuated during the more difficult tracking task. Our results demonstrate that the brain's response to task-irrelevant sensory changes is strongly influenced by intermodal attentional demands.
A model system for addressing such issues has been developed for auditory stimuli. Numerous electrophysiological studies have established that a change in the acoustic properties of a tone that occurs within an otherwise monotonous series of identical tones evokes an event-related potential (ERP) component that occurs approximately $200 \mathrm{msec}$ after the onset of the auditory stimuli. This ERP component has been given the functional label of "mismatch negativity" (MMN) because it is evoked by the tone that does not match the preceding tones in the series and because it is negative in polarity when recorded from scalp electrodes. MMNs are reliably evoked by small changes in tone pitch, duration, intensity, and other more complex stimulus properties (Näätänen \& Winkler, 1999; Näätänen, 1992).

A strong argument has been put forth for the automatic nature of the neural process reflected by the appearance of MMN (Harmony et al., 2000; DittmannBalcar, Thienel, \& Schall, 1999; Woldorff \& Hillyard, 1991; Wickens, Kramer, Vanasse, \& Donchin, 1983; Wickens, 1976); that is, MMN is evoked by deviant tones regardless of the current focus of attention (Atienza \& Cantero, 2001; Fischer, Morlet, \& Giard, 2000). This property has attracted great interest in clinical research where MMN could serve as a marker for a sophisticated neural mechanism whose purpose is to attract attention, but which itself is insensitive to the subject's current attentional state (Näätänen \& Winkler, 1999; Näätänen, 1992). Although an attractive notion, the automaticity of MMN has been challenged. For example, Woldorff and Hillyard (1991) demonstrated that MMN was larger 
when deviant tones were presented to an attended ear compared to the same deviant tone delivered to an unattended ear. Other studies have since demonstrated that MMN is larger when the task-irrelevant deviant tone occurs within the focus of auditory attention (Näätänen, 1992). These studies limit the claims for MMN's automaticity, but confound changes in MMN amplitude with the presence or absence of directed auditory attention to the series of tones containing the irrelevant deviant.

This confound can be overcome by presenting the deviant tones outside of the focus of attention, while manipulating the degree of attention required at the focus. This is easily achieved in intermodal attentional studies where, for example, the attentional demands of a visual task can be varied while the tone series is always task-irrelevant. Dittmann-Balcar et al. (1999) presented subjects with a visual discrimination task at three levels of difficulty and measured MMNs evoked by taskirrelevant deviant tones. No differences in MMN attributable to the difficulty of the visual discrimination were observed in this study, or in a second study by Harmony et al. (2000) that contrasted two levels of visual task difficulty. These results suggest that the auditory mismatch mechanism may be independent of attentional resources devoted to other sensory modalities. However, the discrete and intermittent nature of the stimulus and response characteristics of the visual oddball tasks used by the investigators may have allowed subjects to switch attention rapidly between visual and auditory modalities, and thus, maintain auditory MMN in the face of changing visual demands. During such paradigms attention may be toggled between the visual and auditory channels to simultaneously monitor both. Recent studies have adopted continuous performance tasks, such as continuous visuomotor tracking tasks (Näätänen, Pakarinen, Rinne, \& Takegata, 2004), to enable sustained attentional engagement in the primary visual task while recording neural responses elicited by intermittent unattended deviant tones.

Here we further investigated this issue by engaging subjects in a continuously demanding visuomotor tracking task while recording changes in the neural activation evoked by task-irrelevant deviant tones randomly embedded in a series of identical tone pips. Following Wickens et al. (1983) and Wickens (1976), the attentional demands of the tracking task were varied by changing the control dynamics of the subject's joystick. We conducted one electrophysiological and two neuroimaging studies to measure the brain's response to the deviant tones. These covert measures were then used to determine whether the neural responses evoked by the deviant tones were influenced by variations in the attentional demands of the primary tracking task.

The unique combination of functional magnetic resonance imaging (fMRI) and ERP measures allowed us to resolve both the spatial and the temporal characteristics of change-specific neural responses and their modula- tion by task demands and attention. Through the use of fMRI methods, we sought to determine what brain regions were differentially activated by the task-irrelevant deviant tones compared to the standard tones. Prior electromagnetic field modeling studies have suggested that the MMN component originates in the left and right supratemporal auditory cortices (Escera, Alho, Schroger, \& Winkler, 2000; Alho, 1995; Giard, Lavikainen, et al., 1995; Alho, Huotilainen, et al., 1993; Näätänen, 1992), with additional sources in frontal regions (Muller, Juptner, Jentzen, \& Muller, 2002; Rinne, Alho, Ilmoniemi, Virtanen, \& Näätänen, 2000; Alain, Woods, \& Knight, 1998; Alho, Woods, Algazi, Knight, \& Näätänen, 1994; Giard, Perrin, Pernier, \& Bouchet, 1990) also contributing to the surface field potential. However, fMRI studies alone would not establish how early these brain activations occurred or, indeed, whether the observed hemodynamic changes were related to the MMN. For this reason, we conducted a parallel study with ERP measures. We predicted that for the ERP study, smaller MMN components would be elicited by the deviant tones during the highdifficulty tracking task as compared to the low-difficulty tracking task, reflecting modulation of this component by the attentional demands of the primary task. For the fMRI results, again we predicted that brain activations evoked by deviant tones during the high-difficulty tracking task would be smaller than during the low-difficulty tracking task.

\section{RESULTS}

\section{Behavioral Results}

The results of the preliminary behavioral study confirmed that the second-order visuomotor tracking task was more difficult than the first-order visuomotor tracking task. A repeated-measures ANOVA was performed on the tracking error of the cursor position during the two visual tracking tasks to examine the effects of task difficulty upon tracking performance and $t$ test was used for reaction time measures. The average tracking error was computed for a 3-sec time window preceding each deviant tone, and for a 3-sec time window following each deviant tone in each tracking difficulty. Using second-order dynamics for the joystick controller resulted in significantly greater tracking error of the cursor position $[F(1,10)=170.305, p<.0001]$ and longer reaction times for detecting deviant tones as compared to the first-order control dynamics $[t(10)=$ $-2.24, p<.05$, two-tailed]. Henceforth, we will refer to the second-order tracking condition as high difficulty, and to the first-order tracking condition as low difficulty.

The influence of the deviant tone occurrences on tracking performance was assessed in two fMRI studies and an ERP study separately, where all tones were task irrelevant. As with the behavioral study, there was a significant main effect of control dynamics upon tracking 
error of the cursor position, with second-order dynamics resulting in a larger tracking error than first order in all three studies $[F(1,18)=438.041, p<.0001$ (fMRI Study I) $F(1,14)=224.301, p<.0001$ (fMRI Study II); $F(1,12)=113.125, p<.0001$ (ERP study)]. However, there was no significant difference in the tracking error distance measured before or after the onset of a deviant tone, indicating that the pitch change did not interfere with subjects' performance on the tracking tasks. There were no interaction effects.

\section{Imaging Results}

The task-irrelevant pitch changes evoked activation in temporal regions within and near the superior temporal gyrus (STG), and in the prefrontal cortex. Figures $1 \mathrm{~A}$ and $\mathrm{B}$ illustrate the extent of activation evoked by pitch changes occurring during low- (red hues) and high- (blue hues) difficulty tracking in fMRI Study I. Qualitative differences in the extent of activation are visually apparent with fewer voxels meeting the criteria for significance during high-difficulty tracking. The random-effects analysis results depicted in Figures $1 \mathrm{C}$ and D revealed clusters of activation in the inferior frontal gyrus (IFG) and STG, respectively, for which the hemodynamic responses evoked by irrelevant pitch changes were significantly attenuated during high-difficulty tracking as compared to low-difficulty tracking. In both of these figures, an activation mask consisting of voxels reaching significance $[t(18)=2.5 ; p<.012]$ for either difficulty condition (i.e., all red and all blue voxels) was created and applied to the difference random-effects maps for display purposes to illustrate the differential activation clusters (see Methods). Analysis of the time course within these clusters in the STG further illustrates the larger amplitude of the activation during the low-difficulty tracking condition relative to the high-difficulty tracking condition (Figure 1D).

Analysis of the extent of pitch-deviant tone-dependent activation within the subregions of the STG (Figure 2A) revealed significantly reduced activation in specific loci along the STG for both fMRI Study I (Figure 2B) and fMRI Study II (Figure 2C). This supplemental analysis was thus consistent with the random-effects analysis
Figure 1. Regions exhibiting significant activity in response to pitch-deviant unattended tones during the high-difficulty and low-difficulty tracking tasks in fMRI Study I. (A) Activation maps indicating areas of activation in the STG region (red $=$ low-difficulty tracking task; blue $=$ highdifficulty tracking task); (B) Cross-sectional axial view depicting foci of activity in the temporal cortex and smaller area of activation in the prefrontal cortex $(t \geq 2.5)$; (C) and (D) depict clusters of voxels from the random-effects analysis contrasting the two tracking difficulty conditions (pink $=$ low-difficulty tracking task $>$ high-difficulty tracking task). The time course plots reflect the average BOLD signal change during the two tracking tasks within the depicted random-effects clusters $($ red lines $=$ BOLD response to unattended deviant tone during the lowdifficulty tracking tasks; blue lines $=$ BOLD response to unattended deviant tone during the high-difficulty tracking tasks). The time courses of the average BOLD signal change within the STG clusters reflect the larger response during the low-difficulty tracking task.

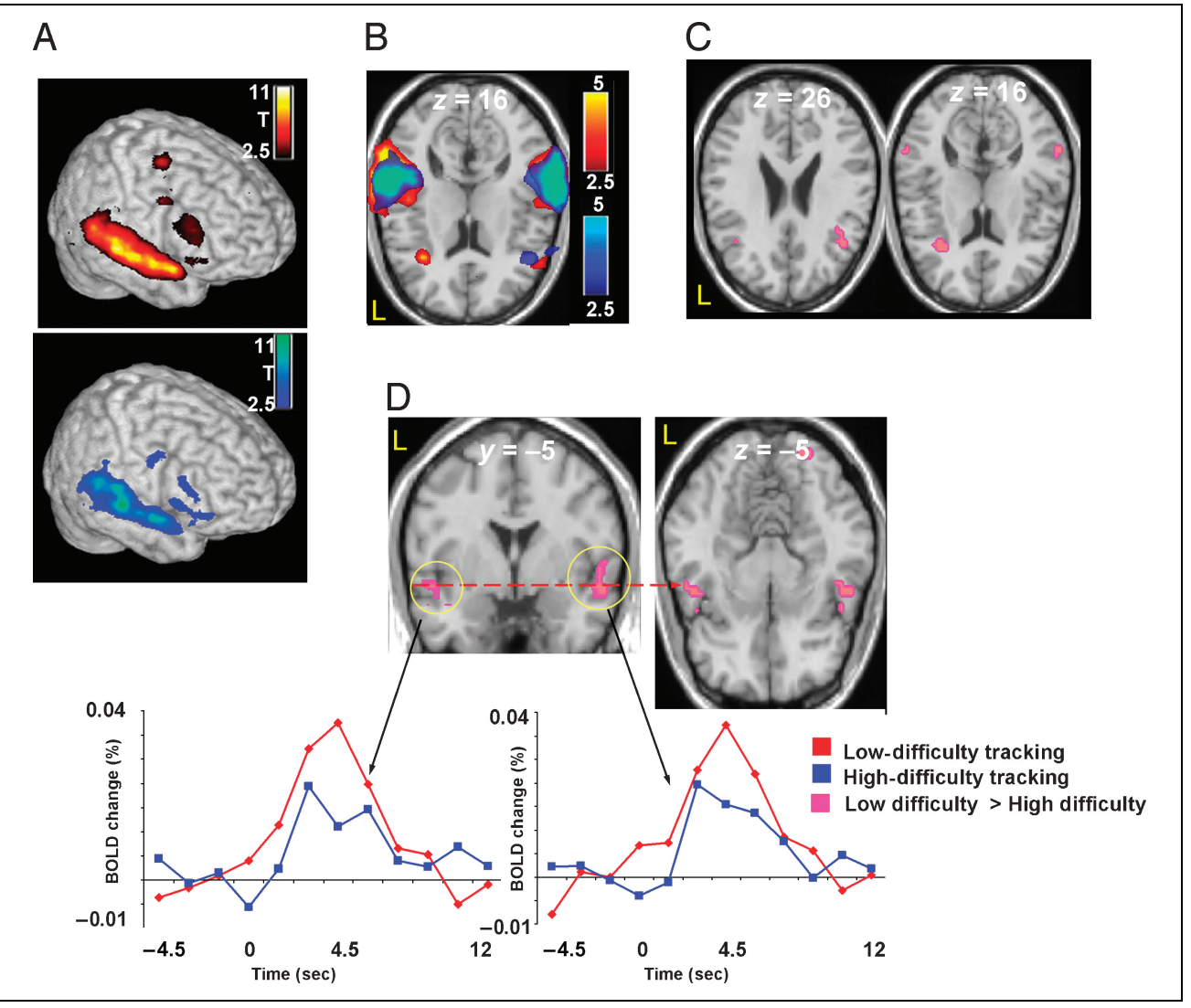


Figure 2. (A) Delineation of the subregions of the STG and the percentage of activated voxels within each subregion during fMRI Study I (B) and fMRI Study II (C) (red = activation to pitch-deviant tones during the low-difficulty tracking task; blue = activation to pitch-deviant tones during the high-difficulty tracking task). (*) indicates $p<.05,(\bullet)$ indicates trend $(p<.06)$.

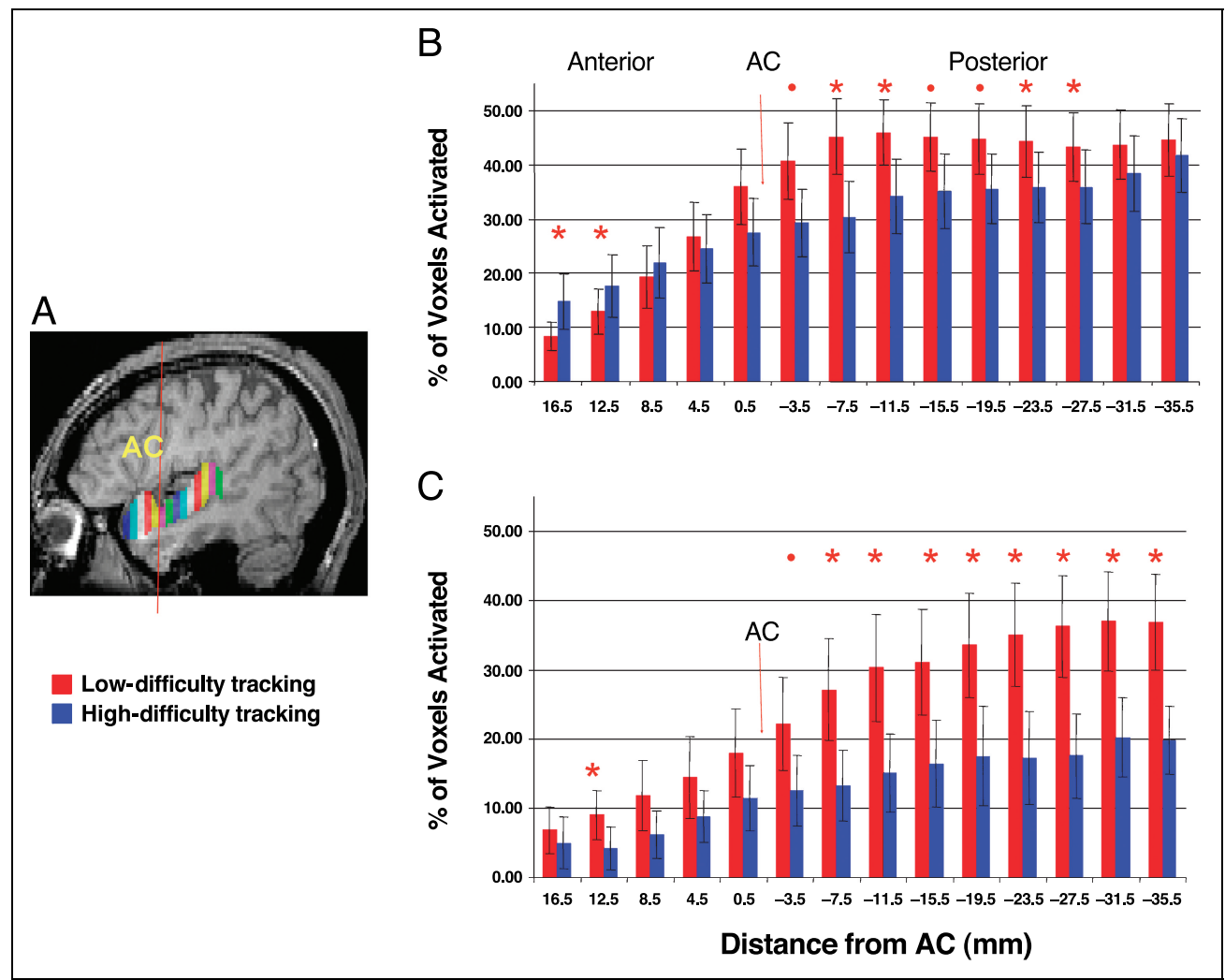

reported in Figures $1 \mathrm{C}$ and D. In contrast to these findings, the amplitude of activation within the Heschl's gyrus (HG) anatomical region of interest (ROI) to pitchdeviant tones during the low- and high-difficulty tracking tasks did not significantly differ.

In order to ensure that the small variations in experimental parameters between fMRI Study I and Study II did not significantly alter the effects of visual tracking complexity on the detection of unattended auditory deviant tones, we conducted a mixed-factor ANOVA on the percent activated voxels in the STG regions to explore interactions between study (fMRI Experiment 1 vs. Experiment 2) and task (low-difficulty vs. high-difficulty tracking) conditions. The results indicated that there was overall more activation to deviant tones in Study I than during Study II (main effect of study, $p<.015$ ) and less activation during the high-difficulty tracking condition in both studies (main effect of tracking, $p<.01$ ). However, there was no significant interaction between study and tracking $(p>.20)$. Thus, for both fMRI studies, the deviant tones elicited greater activation during the low-difficulty visuomotor tracking task as compared to the high-difficulty tracking task.

\section{ERP Results}

To evaluate the influence of tracking task difficulty on auditory-evoked brain responses (including MMN), we recorded ERPs evoked by standard and deviant tones. As typical in ERP studies, the MMN was identified in the difference waveform that resulted from subtracting the ERP evoked by the monotonous standard tones from the ERP evoked by the deviant tones. The topographic map at 180 msec shown in Figure 3A confirms the fronto-central distribution typical for the MMN. As these maps have the same scale, the color difference between the maps obtained during high- and low-difficulty tracking represents the diminished amplitude of MMN during high-difficulty tracking. Repeated-measures ANOVA comparing task (low-difficulty tracking vs. high difficulty tracking) and electrode (F3, Fz, F4) on the MMN mean amplitude showed a significant main effect of task $[F(1,12)=4.803, p=.05]$ and no laterality nor interaction effects. Paired comparisons revealed that the mean amplitudes of the MMN component to deviant tones were significantly smaller during the high-difficulty tracking task at the Fz, F3, and F4 electrode locations $[t(12)=$ $-2.16, p=.026 ; t(12)=-2.35, p=.018 ; t(12)=-2.01$, $p=.033$, respectively, one-tailed]. The amplitude change in MMN at the Fz electrode site can be clearly seen in Figure 3B. The latency-to-peak MMN amplitude at Fz did not differ significantly between the two difficulty levels $[t(12)=-0.857 .35, p=.408$, two-tailed].

\section{DISCUSSION}

The goal of the current study was to examine the attentional modulation of neural activity elicited by task-irrelevant pitch-deviant tones using event-related 


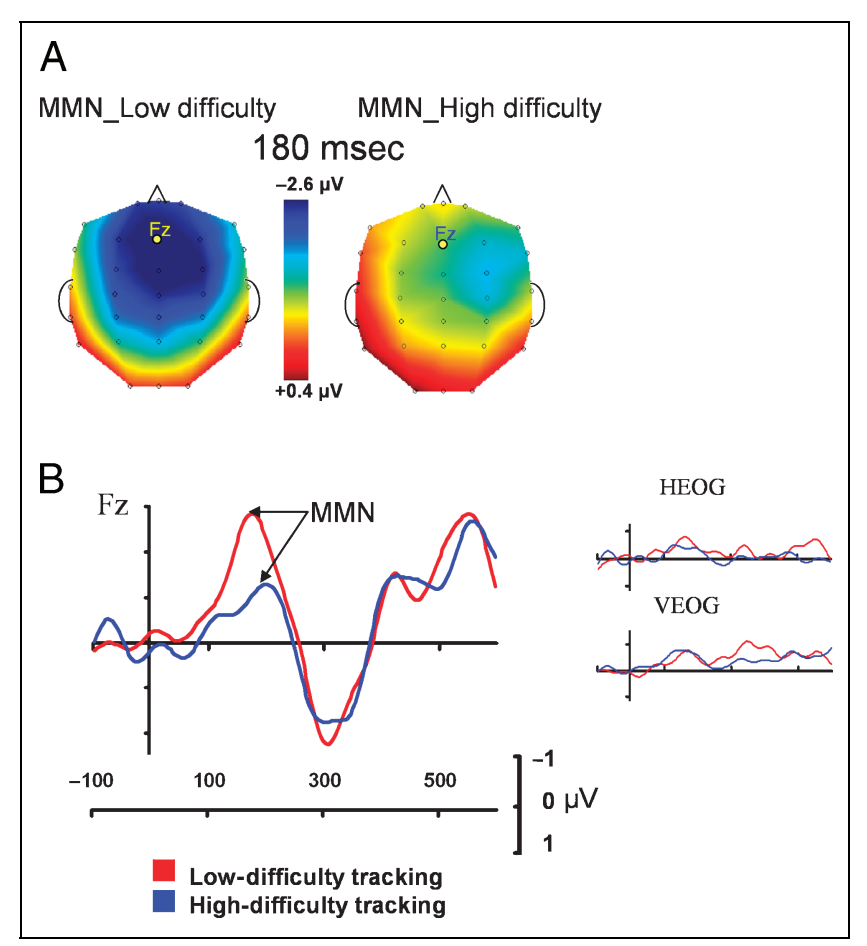

Figure 3. Scalp voltage distribution and amplitude of the MMN (deviant minus standard) component of ERPs. (A) Isovoltage maps show MMN scalp distribution peaking at $180 \mathrm{msec}$ during the two visual tracking tasks. (B) Grand-average difference waves (MMN) ( $n=$ 13 subjects) recorded at the fronto-central electrode (Fz) during the two tracking difficulty levels (red line $=$ MMN amplitude during lowdifficulty tracking task; blue line $=$ MMN amplitude during highdifficulty tracking task). The data were referenced against the chin electrode $(\mathrm{HEOG}=$ grand-average horizontal EOG tracing for low- and high-difficulty tracking conditions; VEOG $=$ grand-average vertical EOG tracings for low- and high-difficulty tracking conditions).

fMRI and ERPs. In agreement with previous imaging studies (Mathiak, Hertrich, Lutzenberger, \& Ackermann, 2002; Opitz, Rinne, Mecklinger, von Cramon, \& Schroger, 2002; Opitz, Mecklinger, Friederici, \& von Cramon, 1999; Opitz, Mecklinger, Von Cramon, \& Kruggel, 1999), unattended deviant tones reliably activated both the frontal and temporal auditory cortex. fMRI results also revealed that the deviant tones elicited significantly smaller and more restricted activation in temporal and frontal regions during the more demanding highdifficulty tracking task relative to the low-difficulty tracking task. Electrophysiological results further supported this finding, in that the auditory MMN was reduced in amplitude during the more difficult visuomotor tracking task. Thus, converging evidence from functional neuroimaging and electrophysiology indicate that the attentional demands of a visuomotor task limits the neural processing afforded to task-irrelevant auditorydeviant tones.

Our ERP result differs from prior studies of MMN that reported no influence of visual task demands upon MMN amplitude (Harmony et al., 2000; Dittmann-Balcar et al., 1999). These prior studies did not use tasks that made continuous demands upon visual attention, and thus the subject may have been able to shift attention between auditory and visual modalities. Our analysis of subject's tracking behavior before and after each auditory deviant suggests that an attention-shifting strategy was not used by our subjects here. We measured no change in moment-to-moment tracking error as a consequence of the occurrence of an auditory deviant.

The fMRI results reported in this study reveal that unattended pitch-deviant tones elicit hemodynamic activity in extensive regions of the STG. This finding is consistent with previous electroencephalographic and magnetoencephalographic source modeling studies of MMN, which have indicated that the auditory MMN component elicited by pitch-deviant tones may be generated in the supratemporal auditory cortex, within and adjacent to the primary auditory cortex (Kropotov et al., 1995; Näätänen \& Alho, 1995). The modulation of these fMRI activations by the attentional demands of the tracking tasks was restricted to a less extensive subregion of the STG (Figures 1C and D). Thus, the attentional demands of the primary task, reflected in the difficulty or error rate of the visuomotor tracking task, attenuated the change-specific responsiveness of the superior temporal cortex. The attentional modulation effect was not found within the HG, which is located on the STG and plays a crucial role in auditory perception. These findings are consistent with a recent study reporting the absence of attentional modulation in the HG (Petkov et al., 2004).

Attentional resource limitations imposed by visuomotor tracking also reduced the activation evoked by auditory deviants in the frontal cortex. Investigators studying the MMN have hypothesized that the frontal sources contributing to the MMN reflect a signal to trigger a shift of attention towards the initially unattended stimuli (Rinne et al., 2000; Alho, 1995). In the inferior frontal regions, the extent of activation to the deviant tones was reduced significantly in the right hemisphere during the high-difficulty tracking task (Figure 2C) as reflected by the random-effects analysis. Attenuation of the frontal hemodynamic response evoked by the deviant tones during the more demanding visual tracking task relative to the low-difficulty visual tracking task suggests that increasing attentional engagement in task-relevant stimuli can reduce the processing of change-specific stimuli in an unattended channel and attentional orienting responses associated with frontal cortical regions.

Recent electrophysiological and magnetoencephalographic recording studies have suggested that changespecific activity in the superior temporal cortex is an objective measure of central auditory processing and can be registered in the absence of attention, with no task requirements (Näätänen et al., 2004; Näätänen, 2003). As such, those studies have proposed that the MMN component of ERPs can be used as a tool for quantifying 
auditory discrimination and sensory memory in numerous clinical populations and very young children. Indeed, the MMN component has been recorded in sleeping newborn babies (Alho, Sainio, Sajaniemi, Reinikainen, \& Näätänen, 1990). Thus, the MMN has been proposed as an early screening tool for neurodevelopmental disorders accompanied by deficits in the perception of speech sounds (Leppèanen \& Lyytinen, 1997). The MMN has also been recently used to explore phonological and auditory processing deficits in dyslexic adolescents (Baldeweg, Richardson, Watkins, Foale, \& Gruzelier, 1999; Schulte-Kèorne, Deimel, Bartling, \& Remschmidt, 1998). Deficits in auditory change-specific measures have also been reported in schizophrenia (Umbricht et al., 2000; Shelley et al., 1991), and have been interpreted as reflecting central auditory cortex pathology associated with NMDA-receptor dysfunction (Javitt, Shelley, Silipo, \& Lieberman, 2000).

Our findings suggest that the MMN should be applied cautiously as an objective clinical measure in that these neural responses can be significantly attenuated as a function of primary task difficulty. Our fMRI findings further demonstrate that both frontal and temporal cortical responses to deviant tones are attenuated during demanding continuous performance tasks. As the pathophysiology of many neuropsychiatric and neurodevelopmental disorders include significant frontal cortical involvement, it is imperative to examine whether any deficit in the auditory change-specific neural processes occur independently of changes in the primary task demands and attributes that may rely upon frontal cortical circuits. Designing experimental paradigms that manipulate the demands and attributes of the primary task would confer experimenters and clinicians the ability to better interpret the source of recorded MMN components or change-specific neural activity deficits.

\section{Conclusion}

In summary, the present results are compatible with prevailing cognitive theory and provide new insight into the evolution of activation in temporal and frontal cortical areas that are specific to involuntary attention to task-irrelevant auditory stimuli. The results of this study demonstrate that when the demands of a primary task are increased-the portion of capacity used by that process rises-there is a corresponding decrease in neural and hemodynamic activity to task-irrelevant sensory changes in both sensory and association cortices. This study reveals that neural activity in fronto-temporal, higher-order processing regions are modulated by attention, and thus, suggest that these regions subserve the selection of task-relevant stimuli and the filtering out of task-irrelevant potentially interfering stimuli. In contrast, the primary auditory cortex (HG) was insensitive to our attentional manipulation, hence, may enable faithful monitoring of sensory information and its reli- able transfer to higher-order processing regions. A model where attention modulates the neural responses elicited by unattended auditory-deviant tones would predict that stimulus salience or altered global attentional capacity associated with clinical states may affect the amplitude and extent of the change-specific neural responses to unattended auditory stimuli.

\section{METHODS}

\section{Subjects}

In this article, we report the results of a behavioral experiment, two fMRI experiments, and an ERP experiment. Each of these experiments was conducted separately with different groups of subjects. Each experiment was approved by the Duke University Institutional Review Board and all subjects provided informed consent. All subjects reported normal hearing and (corrected-to) normal visual acuity, and none reported serious neurological or psychiatric problems. All subjects were right-handed and handedness was confirmed by the Edinburgh Handedness Questionnaire (Oldfield, 1971).

Data from 11 of 12 subjects (age range 18-23 years, mean age $=19$ years; 6 women) were included in the behavioral analyses. One subject was excluded because of a technical problem. Data from 19 of 25 subjects (age range $19-32$ years, mean age $=22$ years; 10 women) were included in fMRI Study I analyses. Data from 14 of 15 subjects (age range $19-33$ years, mean age $=23$ years; 9 women) were included in fMRI Study II analyses. Six subjects in fMRI Study I and one subject in fMRI Study II were excluded due to head motion of greater than one voxel size. Data from 13 of 17 subjects (age range $19-28$ years, mean age $=22$ years; 8 women) were included in the ERP analyses. Four subjects were discarded due to excessive eye movement artifacts or technical problems.

\section{Experimental Task}

We implemented a continuous visuomotor tracking task in which subjects operated an isometric joystick to center a small cursor on a visual display. The cursor's position was perturbed by a two-dimensional forcing function that consisted of band-limited noise with brief large deviations or steps superimposed. Following prior studies by Wickens et al. (1983), we created two difficulty levels by altering the dynamics of the joystick. Firstorder (velocity) dynamics were used for the lower level of difficulty. Second-order (acceleration) dynamics were used for the higher level of difficulty. Tracking error was quantified as the Euclidian distance between the cursor's position and the small fixation cross at the center of the display. Tracking error was continuously sampled at $10 \mathrm{~Hz}$ and stored for off-line analysis. This permitted an analysis of short-term changes in tracking performance 
that may have been related to the presentation of auditory tones.

Auditory tone pips were presented concurrently with visuomotor tracking. The auditory tones were delivered at a constant rate of one tone per $1500 \mathrm{msec}$. The duration of each tone was $200 \mathrm{msec}$ (with $10 \mathrm{msec}$ rise/fall times) and was presented at $85 \mathrm{~dB}$ SPL through headphones. In fMRI Study I and the ERP study, two tone frequencies were used. The standard tones were $600 \mathrm{~Hz}$ and occurred on $\sim 92 \%$ of trials. The deviant tones were $780 \mathrm{~Hz}$ and occurred on $~ 8 \%$ of trials with an interval randomized between 12 and 21 sec separating successive deviants. In fMRI Study II, three tone frequencies were used. The standard tones were $600 \mathrm{~Hz}$ and occurred on $89 \%$ of trials. The deviant ones were 514 and $700 \mathrm{~Hz}$ (50\% each) and occurred on 11\% of trials, with an interval randomized between 9 and $15 \mathrm{sec}$ separating successive deviants.

Subjects in the preliminary behavioral study were required to depress a button on the joystick whenever they detected a deviant tone. This manipulation was used to validate that visuomotor tracking difficulty influenced reaction time to the deviants. In both the fMRI and ERP experiments, the tones were always taskirrelevant and subjects were instructed to ignore them. In both fMRI experiments, subjects performed three runs of low-difficulty tracking and three runs of highdifficulty tracking. During fMRI Study II, both deviant tones were randomly presented during each run. Tracking task difficulty order was randomized across runs. During fMRI Study II, activations from the two pitchdeviant tones were combined as a deviant stimulus type and analyzed with the same procedure as fMRI Study I (see Image Analysis). Each of the six runs lasted $\sim 5.25 \mathrm{~min}$, during which time 210 auditory tones were presented. Prior to scanning, a practice run of $\sim 75 \mathrm{sec}$ was conducted to familiarize the subjects with the task. In the ERP experiment, subjects performed eight runs of low-difficulty tracking and eight runs of high-difficulty tracking in random order. Each run lasted $\sim 3.4 \mathrm{~min}$ during which time 135 auditory stimuli were presented.

\section{Image Acquisition}

Scanning was performed on a GE 4T LX Nvi MRI scanner with $41 \mathrm{mT} / \mathrm{m}$ gradients (General Electric, Milwaukee, WI) and a birdcage RF head coil. The anterior commissure (AC) and posterior commissure (PC) were identified in a sagittal T1-weighted localizer series, and all subsequent series were acquired in a near-axial plane parallel to the AC and PC. A 3-D IR-prepped SPGR series (repetition time $[\mathrm{TR}]=12.3 \mathrm{msec}$; echo time $[\mathrm{TE}]=$ $5.3 \mathrm{msec}$; field of view $[\mathrm{FOV}]=24 \mathrm{~cm}$; image matrix $=$ $256 \times 256$; slice thickness $=1.9 \mathrm{~mm}$, in-plane resolution $=0.9375 \mathrm{~mm}^{2}$; inversion time $\mathrm{T} 1=300 \mathrm{msec}$ ) consisting of 68 slices were used for normalization and co-registration with functional images.
Gradient-echo images $(\mathrm{TR}=1500 \mathrm{msec} ; \mathrm{TE}=31 \mathrm{msec}$; FOV $=24 \mathrm{~cm}$; image matrix $=64^{2}$; flip angle $=60^{\circ}$; slice thickness $=3.8 \mathrm{~mm}$; in-plane resolution $=3.75 \mathrm{~mm}^{2}$ ) sensitive to blood oxygen level dependent (BOLD) contrast were acquired using a spiral imaging $k$-space trajectory. Thirty-four slices were acquired per volume providing whole-brain coverage at near-isotropic resolution. Each imaging run began with four discarded RF excitations to allow for steady-state equilibrium. A semiautomated high-order shimming program was used before functional image acquisition.

Participants viewed the visuomotor tracking display through LCD goggles while tones were presented through MRI-compatible headphones (Resonance Technology, Northridge, CA).

\section{Image Analysis}

Head motion was detected by center of mass measurements. The data of seven subjects were discarded because these subjects had greater than a $1.5-\mathrm{mm}$ deviation in the center of mass in any plane. The Statistical Parametric Mapping (SPM99) analysis software (The Welcome Department of Cognitive Neurology, London) was used to correct for timing differences due to slice acquisition order, for normalization to a standard stereotaxic space (Montreal Neurological Institute brain template), and for smoothing ( $8 \mathrm{~mm}$ FWHM) prior to voxel-based analysis. Additional analyses were conducted using custom software. Short time series of image volumes time-locked to the onset of the deviant tone were extracted from the continuous time series and averaged in separate bins for low- and high-difficulty tracking conditions. Epochs with image time points that showed intensity shifts that varied by 3 standard deviations above or below the mean were excluded from the epoch averaging. This procedure eliminated a maximum of 5 epochs for each bin condition for each subject. The remaining bin-averaged epochs consisted of a total of 12 image volumes that included four baseline volumes $(-4.5$ to $0 \mathrm{sec})$ prior to the deviant tone onset and 10 image volumes (0 to $12 \mathrm{sec}$ ) after deviant onset. The voxel-by-voxel means of the four baseline volumes were subtracted from each volume, and so the poststimulus portions of the epochs represented MRI signal deviation evoked by the deviant tone. The mean baseline volume was retained so that these deviations could be easily converted to percent signal changes.

The average time course of activity evoked at each voxel for each tracking difficulty condition and for each subject was correlated with an empirical hemodynamic reference waveform to identify those voxels activated by the deviant tones. Random-effects analyses were performed on these voxels to identify regions where activity for each task condition exceeded baseline activity at $t=2.5$ significance level. Figures $1 \mathrm{~A}$ and $\mathrm{B}$ represent the average $t$-statistics produced by this analysis. 
To further identify regions that were differentially activated by the pitch-deviant tones during the two tracking difficulty conditions, a time-series volume was computed for each voxel for each subject at each time point in the epoch representing differential signal change between the two conditions.

A random-effects analysis was performed on the $t$-statistics generated for each subject at each voxel. This analysis was restricted to the 3-7.5 sec portion of the poststimulus epoch to capture the peak of the hemodynamic response. Although this voxel-based randomeffects analysis comparing the influence of task difficulty was conducted on all voxels, only voxels within the masked region (i.e., voxels significantly activated by individual task conditions at $t=2.5$ as described above) depicting the differential activation between tracking conditions at a significance level of $t=2$ were displayed in Figures $1 \mathrm{C}$ and D. The hemodynamic responses shown in Figure 1 represent the percent signal change within the activation from the STG as shown in Figure 1D.

Finally, an anatomical ROI analysis was performed on the STG and HG. This supplementary analysis compensated for possible misregistration errors in the voxelbased analyses performed on normalized images. Based upon the extent literature (Opitz, Rinne, et al., 2002; Opitz, Mecklinger, Friederici, et al., 1999; Giard, Perrin, et al., 1990), these ROIs were traced manually by identifying the anatomical boundaries of the left and right STG and using IRIS/SNAP software (Ho, Bullit, \& Gerig, 2002), guided by human brain atlases (Duvernoy, 1999) and the LONI guidelines (Laboratory of Neuro Imaging Resources, Sulcal Guidelines). We subsequently divided the STG volume into subregions extending from $16 \mathrm{~mm}$ anterior to $35.5 \mathrm{~mm}$ posterior to the $\mathrm{AC}$ in $3.5 \mathrm{~mm}$ sections, representing functional voxel size as depicted by Figure 2A. ROIs were further restricted to voxels within each of these anatomical regions that were found to be significantly active at a level of $t=2$. At greater $t$-value thresholds, the difference between the tracking conditions increased, hence, we selected this threshold to obtain a conservative estimate of the overall task differences in these regions. Statistical analyses were performed to compare the extent of activation within each of these subregions for fMRI Study I (Figure 2B) and fMRI Study II (Figure 2C). Finally, to examine taskdependent modulation of pitch-deviant tone activation in the primary auditory cortex specifically, we manually traced the anatomical boundaries of the HG again according to LONI guidelines and compared the extent and amplitude of the HDR in this region under the two tracking difficulty levels.

\section{ERP Recording and Analysis}

The EEG was recorded from an array of 31 tin electrodes attached to fabric caps (Electro-Cap International, Eaton, $\mathrm{OH})$. The array included the locations of the
10-20 system, and include two electrodes placed at the outer canthi and below of the right eye to record the electrooculogram (EOG). Electrode impedances were kept below $5 \mathrm{k} \Omega$. All sites were referenced to the chin electrode. The EEG was amplified with a gain of 20,000 through a band pass of $0.01-100 \mathrm{~Hz}$, digitized at $250 \mathrm{~Hz}$ per channel, and stored for off-line analysis.

Epochs of $700 \mathrm{msec}$ length (including $100 \mathrm{msec}$ prestimulus baseline) were extracted from the continuous EEG recordings and then averaged into separate bins for each subject, electrode, tracking difficulty condition, and stimulus type (standard or deviant). Epochs contaminated with EOG artifacts were detected by an automated algorithm and excluded from the bin averaging procedure. The resulting averaged ERPs were low-pass filtered $(15 \mathrm{~Hz})$. The mean voltage of the $100 \mathrm{msec}$ prestimulus period served as a baseline for amplitude measurement.

Difference waveforms were created at each electrode site representing the difference between the ERPs evoked by the standard and deviant tones. Such difference waveforms have been used in many prior studies to isolate the broad negative ERP component referred to as the MMN. Color-coded isovoltage maps were created to visualize the distribution of MMN across the scalp array so that our recordings could be compared to the typical distribution obtained for MMN. Because the MMN is typically maximal at fronto-central electrode locations (Näätänen \& Winkler, 1999), we selected the F3, Fz, and F4 electrode sites for statistical analysis. The MMN peak latencies were measured from the most negative peak occurring at the 120-250 msec poststimulus period. The MMN amplitudes were calculated as a mean voltage at the 40-msec period centered at the peak latency in the averaged data for each subject.

\section{Acknowledgments}

This research was supported by NINDS grant NS41328. Gregory McCarthy was supported by a DVA Senior Career Research Scientist award. We thank Charles Michelich and Joshua Bizzell for assistance in data analysis and Jeff Hoerle for programming the tasks as well as Dr. Benjamin Chen and Jordan Tozer for assistance with scanning.

Reprint requests should be sent to Aysenil Belger, The DukeUNC Brain Imaging and Analysis Center, Box 3918, Duke University Medical Center, Durham, NC 27710, or via e-mail: abelger@med.unc.edu.

The data reported in this experiment have been deposited with the fMRI Data Center archive (www.fmridc.org). The accession number is 2-2005-1192B.

\section{REFERENCES}

Alain, C., Woods, D. L., \& Knight, R. T. (1998). A distributed cortical network for auditory sensory memory in humans. Brain Research, 812, 23-37.

Alho, K. (1995). Cerebral generators of mismatch negativity $(\mathrm{MMN})$ and its magnetic counterpart (mMNM) elicited by sound changes. Ear \& Hearing, 16, 38-51. 
Alho, K., Huotilainen, M., Tiitinen, H., Ilmoniemi, R. J., Knuutila, J., \& Näätänen, R. (1993). Memory-related processing of complex sound patterns in human auditory cortex: A MEG study. NeuroReport, 4, 391-394.

Alho, K., Medvedev, S. V., Pakhomov, S. V., Roudas, M. S., Tervaniemi, M., Reinikainen, K., Zeffiro T., \& Näätänen R. (1999). Selective tuning of the left and right auditory cortices during spatially directed attention. Brain Research, Cognitive Brain Research, 7, 335-341.

Alho, K., Sainio, K., Sajaniemi, N., Reinikainen, K., \& Näätänen, R. (1990). Event-related brain potential of human newborns to pitch change of an acoustic stimulus. Electroencephalography and Clinical Neurophysiology, 77, 151-155.

Alho, K., Woods, D. L., Algazi, A., Knight, R. T., \& Näätänen, R. (1994). Lesions of frontal cortex diminish the auditory mismatch negativity. Electroencephalography and Clinical Neurophysiology, 91, 353-362.

Alho, K., Woods, D. L., Algazi, A., \& Näätänen, R. (1992). Intermodal selective attention: II. Effects of attentional load on processing of auditory and visual stimuli in central space. Electroencephalography and Clinical Neurophysiology, 82, 356-368.

Arnott, S. R., \& Alain, C. (2002). Stepping out of the spotlight: MMN attenuation as a function of distance from the attended location. NeuroReport, 13, 2209-2212.

Atienza, M., \& Cantero, J. L. (2001). Complex sound processing during human REM sleep by recovering information from long-term memory as revealed by the mismatch negativity (mmn). Brain Research, 901, 151-160.

Baldeweg, T., Richardson, A., Watkins, S., Foale, C., \& Gruzelier, J. (1999). Impaired auditory frequency discrimination in dyslexia detected with mismatch evoked potentials. Annals of Neurology, 45, 495-503.

Dittmann-Balcar, A., Thienel, R., \& Schall, U. (1999). Attention-dependent allocation of auditory processing resources as measured by mismatch negativity. NeuroReport, 10, 3749-3753.

Duvernoy, H. M. (1999). The human brain: Surface, three-dimensional sectional anatomy with MRI, and blood supply (2nd ed.). New York: Springer-Verlag Wien.

Escera, C., Alho, K., Schroger, E., \& Winkler, I. (2000). Involuntary attention and distractibility as evaluated with event-related brain potentials. Audiology \& Neuro-Otology, 5, 151-166.

Fischer, C., Morlet, D., \& Giard, M. (2000). Mismatch negativity and n100 in comatose patients. Audiology \& Neuro-Otology, 5, 192-197.

Giard, M. H., Lavikainen, J., Reinikainen, K., Perrin, F., Bertrand, O., Pernier, J., \& Näätänen, R. (1995). Separate representation of stimulus frequency, intensity, and duration in auditory sensory memory: An event-related potential and dipole-model analysis. Journal of Cognitive Neuroscience, 7, 133-143.

Giard, M. H., Perrin, F., Pernier, J., \& Bouchet, P. (1990). Brain generators implicated in the processing of auditory stimulus deviance: A topographic event-related potential study. Psychophysiology, 27, 627-640.

Harmony, T., Bernal, J., Fernandez, T., Silva-Pereyra, J., Fernandez-Bouzas, A., Marosi, E., Rodriguez, M., \& Reyes, A. (2000). Primary task demands modulate P3a amplitude. Brain Research, Cognitive Brain Research, 9, 53-60.

Hillyard, S. A., Teder-Salejarvi, W. A., \& Munte, T. F. (1998). Temporal dynamics of early perceptual processing. Current Opinion in Neurobiology, 8, 202-210.

Ho, S., Bullit, E., \& Gerig, G. (2002). Level set evolution with region competition: Automatic 3-D segmentation of brain tumors. Paper presented at the 16th International
Conference on Pattern Recognition ICPR, IEEE Computer Society.

Javitt, D. C., Shelley, A. M., Silipo, G., \& Lieberman, J. A. (2000). Deficits in auditory and visual context-dependent processing in schizophrenia: Defining the pattern. Archives of General Psychiatry, 57, 1131-1137.

Kropotov, J. D., Näätänen, R., Sevostianov, A. V., Alho, K., Reinikainen, K., \& Kropotova, O. V. (1995). Mismatch negativity to auditory stimulus change recorded directly from the human temporal cortex. Psychophysiology, 32, 418-422.

Leppèanen, P. H., \& Lyytinen, H. (1997). Auditory event-related potentials in the study of developmental language-related disorders. Audiology \& Neuro-Otology, 2, 308-340.

Mathiak, K., Hertrich, I., Lutzenberger, W., \& Ackermann, H. (2002). Functional cerebral asymmetries of pitch processing during dichotic stimulus application: A whole-head magnetoencephalography study. Neuropsychologia, 40, 585-593.

Muller, B. W., Juptner, M., Jentzen, W., \& Muller, S. P. (2002). Cortical activation to auditory mismatch elicited by frequency deviant and complex novel sounds: A PET study. Neuroimage, 17, 231-239.

Näätänen, R. (1992). Attention and brain function. Hillsdale, NJ: Erlbaum.

Näätänen, R. (2003). Mismatch negativity: Clinical research and possible applications. International Journal of Psychophysiology, 48, 179-188.

Näätänen, R., \& Alho, K. (1995). Mismatch negativity-A unique measure of sensory processing in audition. International Journal of Neuroscience, 80, 317-337.

Näätänen, R., Pakarinen, S., Rinne, T., \& Takegata, R. (2004). The mismatch negativity $(\mathrm{mmn})$ : Towards the optimal paradigm. Clinical Neurophysiology, 115, 140-144.

Näätänen, R., \& Winkler, I. (1999). The concept of auditory stimulus representation in cognitive neuroscience. Psychological Bulletin, 125, 826-859.

Oldfield, R. C. (1971). The assessment and analysis of handedness: The Edinburgh Inventory. Neuropsychologia, 9, 97-113.

Opitz, B., Mecklinger, A., Friederici, A. D., \& von Cramon, D. Y. (1999). The functional neuroanatomy of novelty processing: Integrating ERP and fMRI results. Cerebral Cortex, 9, 379-391.

Opitz, B., Mecklinger, A., Von Cramon, D. Y., \& Kruggel, F. (1999). Combining electrophysiological and hemodynamic measures of the auditory oddball. Psychophysiology, 36, 142-147.

Opitz, B., Rinne, T., Mecklinger, A., von Cramon, D. Y., \& Schroger, E. (2002). Differential contribution of frontal and temporal cortices to auditory change detection: fMRI and ERP results. Neuroimage, 15, 167-174.

Petkov, C. I., Kang, X., Alho, K., Bertrand, O., Yund, E. W., \& Woods, D. L. (2004). Attentional modulation of human auditory cortex. Nature Neuroscience, 7, 658-663.

Rinne, T., Alho, K., Ilmoniemi, R. J., Virtanen, J., \& Näätänen, R. (2000). Separate time behaviors of the temporal and frontal mismatch negativity sources. Neuroimage, 12, 14-19.

Schulte-Kèorne, G., Deimel, W., Bartling, J., \& Remschmidt, H. (1998). Auditory processing and dyslexia: Evidence for a specific speech processing deficit. NeuroReport, 9, 337-340.

Shelley, A. M., Ward, P. B., Catts, S. V., Michie, P. T., Andrews, S., \& McConaghy, N. (1991). Mismatch negativity: An index of a preattentive processing deficit in schizophrenia. Biological Psychiatry, 30, 1059-1062.

Spence, C., Ranson, J., \& Driver, J. (2000). Cross-modal selective attention: On the difficulty of ignoring sounds 
at the locus of visual attention. Perception E Psychophysics, 62, 410-424.

Umbricht, D., Schmid, L., Koller, R., Vollenweider, F. X., Hell, D., \& Javitt, D. C. (2000). Ketamine-induced deficits in auditory and visual context-dependent processing in healthy volunteers: Implications for models of cognitive deficits in schizophrenia. Archives of General Psychiatry, 57, 1139-1147.

Wickens, C., Kramer, A., Vanasse, L., \& Donchin, E. (1983). Performance of concurrent tasks: A psychophysiological analysis of the reciprocity of information-processing resources. Science, 221, 1080-1082.

Wickens, C. D. (1976). The effects of divided attention on information processing in manual tracking. Journal of
Experimental Psychology: Human Perception and Performance, 2, 1-13.

Woldorff, M. G., \& Hillyard, S. A. (1991). Modulation of early auditory processing during selective listening to rapidly presented tones. Electroencephalography and Clinical Neurophysiology, 79, 170-191.

Woodruff, P. W. (1996). Modulation of auditory and visual cortex by selective attention is modality-dependent. NeuroReport, 7, 1909-1913.

Woods, D. L., Alho, K., \& Algazi, A. (1992). Intermodal selective attention: I. Effects on event-related potentials to lateralized auditory and visual stimuli. Electroencephalography and Clinical Neurophysiology, 82, 341-355. 